\title{
Sensitivity analysis for feedforward control system design
}

\author{
Héctor M. Rodríguez and Ricardo A. Burdisso \\ Vibration and Acoustics Laboratories, Department of Mechanical Engineering, Virginia Polytechnic \\ Institute and State University, Blacksburg, Virginia 24061-0238
}

(Received 27 September 1994; revised 4 May 1995; accepted 7 August 1995)

\begin{abstract}
A sensitivity analysis for feedforward-controlled finite domain systems is presented. Using an analytical approach, the sensitivity of a controlled system to variations in the design variables of the control inputs and the error sensors is obtained. Since the physical implementation of the control inputs and the error sensors is directly related to the modal control forces and the modal error sensor components, the analysis is performed in the modal domain. This modal domain analysis offers the advantage that the highest computational effort in obtaining the sensitivities is independent of the physical characteristics of the transducers. From the results in the modal domain, the sensitivities of the controlled system with respect to particular physical parameters are obtained by using the chain rule of differentiation. Therefore, different types of transducers can be investigated within a minimum computational cost. The formulation can be easily incorporated into an optimization procedure for the optimal design of feedforward control systems. A numerical example in which the proposed formulation is compared to results obtained using finite differences is included. (C) 1995 Acoustical Society of America.
\end{abstract}

PACS numbers: $43.40 . \mathrm{Vn}$

\section{INTRODUCTION}

In feedfoward control approaches the undesirable response due to a persistent disturbance is reduced by applying control inputs such that some error information is minimized. The control inputs are obtained by "feeding forward" fully coherent information from the disturbing signal through the control compensator. The optimum control inputs are obtained by minimizing a quadratic cost function of the response to be attenuated. ${ }^{1}$ The sum of the mean-square-value signal from an array of sensors on the structure or in the acoustic field are commonly used to obtain the error information to be minimized.

Recent advances in digital signal processing, as well as new actuator and sensor materials, have made possible the implementation of this control approach in real systems in which the excitation signal is stationary, i.e., pure tone, multiple frequencies, and random. At this time, feedforward control approaches have proven to be successful for active noise control (ANC) applications. ${ }^{1}$ It has also been extensively used in active structural acoustic control (ASAC) applications ${ }^{2-4}$ where the sound field generated by a vibrating structure is attenuated by applying control inputs directly on the structure. For the control of structural vibration (AVC), it has been successfully applied to reduce bending and extensional waves on infinite or semi-infinite structures. ${ }^{5}$

The design of feedforward control systems involves the proper selection of transducer parameters such as location, shape distribution, configuration, and so forth. Previous work has demonstrated that the use of formal optimization approaches during the design process can have a profound impact on the performance of such systems. ${ }^{6,7}$ In particular, it was shown that properly located transducers can reduce the dimensionality and complexity of the controller. ${ }^{7}$ The optimum design variables are obtained by minimizing a function that is, in general, different from the function used to obtain the optimum control inputs. For the sake of clarity, the function used to obtain the optimum design parameters of the control inputs and error sensors will be referred here as the performance function, while the function used to determine optimum control inputs is termed the control cost function. The average mean-square vibration level over the structure and the total radiated power are typical examples of performance functions for vibration and radiation control, respectively.

Optimization algorithms involve the use of sensitivity (gradient) information in its search for an optimum solution. This task can be easily performed using numerical techniques such as the finite difference method (FDM). The main disadvantage of the FDM is that the number of function evaluations increases significantly making an optimization algorithm inefficient. In addition, it often has accuracy problems. ${ }^{8,9}$

In this paper, a formulation is presented to compute the sensitivities of feedforward-controlled systems to variations in the design variables related to the configurations of the control inputs and the error sensors. The formulation is specifically applicable to problems where the response of the system can be expressed as a linear combination of modes, i.e., finite domain systems. When the response is expressed in the modal domain, the physical characteristics of the actuators and sensors are directly related to the modal control forces and modal error sensor components, respectively. Therefore, the formulation is derived in the modal domain. The sensitivity with respect to the physical design parameters of a particular transducer can be easily obtained by using the chain rule of differentiation. A numerical example where the proposed formulation is compared to the central differences approach of the FDM is included. In the example, the finite difference approximation is computed using a range of different perturbations. From the results, it is 


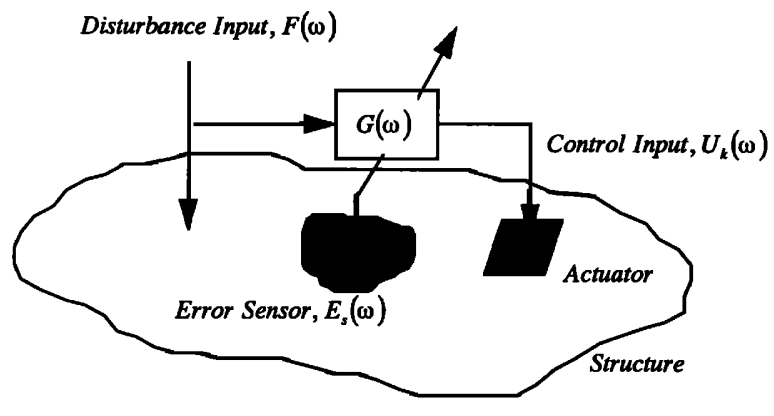

FIG. 1. Typical feedforward control arrangement.

shown that the proposed formulation presents an effective method to compute the desired sensitivities, providing the accuracy of an analytical solution with a minimum computational cost.

\section{FEEDFORWARD CONTROL}

In feedforward control, the response of a system due to an input disturbance $F(\omega)$ is reduced by applying secondary control inputs $U_{k}(\omega)$ as shown in Fig. 1. As mentioned before, the control inputs are obtained by feeding forward a reference signal fully coherent to the input disturbance into the compensators $G(\omega)$. The compensators are designed such that the output from the error sensors $E_{s}(\omega)$ is minimized. Assuming that the disturbance input is stationary, the analysis can be performed in the frequency domain by taking the Fourier transform of any time-dependent variable.

\section{A. System response}

In structural applications the input disturbance can be viewed as a force producing an undesirable response, and the control inputs as forces applied to the structure to minimize that response. Assuming stationarity of both the disturbance and the control forces, the response in the frequency domain can be obtained as a linear combination of the modes as

$$
w(D, \omega)=\sum_{n=1}^{N} q_{n}(\omega) \phi_{n}(D),
$$

where $w(D, \omega)$ is the displacement in the structural domain $D$ at a frequency $\omega, q_{n}(\omega)$ is the Fourier transform of the $n$th modal displacement, $\phi_{n}(D)$ is the $n$th mode shape, and $N$ is the total number of modes included in the analysis. The modal displacement $q_{n}(\omega)$ is expressed as

$$
q_{n}(\omega)=\left(f_{n} F(\omega)+\sum_{k=1}^{N_{c}} u_{n k} U_{k}(\omega)\right) H_{n}(\omega),
$$

where $F(\omega)$ and $U_{k}(\omega)$ are the complex amplitudes of the disturbance and the $k$ th control input, respectively; $f_{n}$ and $u_{n k}$ are the $n$th unit modal forces due to the input disturbance and the $k$ th modal control force, respectively; and $N_{c}$ is the total number of control inputs. The modal quantities $f_{n}$ and $u_{n k}$ are related to the physical implementation of the disturbance and the control inputs. For example, point forces will result in the unit modal forces taking the value of the mode shape at the force location. Similarly, distributed strain induced actuators will yield modal parameters related to the first derivative of the mode shapes. ${ }^{10}$ Finally, $H_{n}(\omega)$ is the $n$th modal frequency response function which is defined as

$$
H_{n}(\omega)=\frac{1}{\omega_{n}^{2}-\omega^{2}+2 j \beta_{n} \omega_{n} \omega},
$$

where $\omega_{n}$ is the $n$th natural frequency, $\beta_{n}$ is the $n$th modal damping ratio, and $j$ is the imaginary number. Equation (2) assumes the mode shapes are normalized with respect to the mass distribution. Equation (1) represents the controlled response of a vibrating structure. The uncontrolled response is obtained by simply setting the control inputs $U_{k}(\omega)$ to zero in Eq. (2).

\section{B. Optimum control inputs}

The complex amplitudes of the control inputs $U_{k}(\omega)$ are obtained by minimizing a quadratic cost function of a measurable response quantity. ${ }^{1}$ This cost function, which is referred here as the control cost function, is defined as the sum of the mean-square value (msv) of the error sensor outputs. That is

$$
C=\sum_{s=1}^{N_{s}} \int_{-\infty}^{\infty} E_{s}(\omega) E_{s}^{*}(\omega) d \omega
$$

where $E_{s}(\omega)$ is the Fourier transform of the $s$ th error sensor output, $N_{s}$ is the total number of error sensors, and (*) denotes the complex conjugate.

Here it is assumed that only structural error transducers are considered. Thus the error variable can always be expressed as a linear combination of modal terms as ${ }^{11,12}$

$$
E_{s}(\omega)=\sum_{n=1}^{N} q_{n}(\omega) \xi_{n s}
$$

where $\xi_{n s}$ is the $n$th modal error component of the $s$ th error sensor. Again, the modal error components are related to the physical implementation of the transducers. A discrete displacement sensor will yield a modal error component that is the value of the mode shape at the sensor location.

As it is shown in the literature, the control cost function in Eq. (4) is a positive definite function with respect to $U_{k}(\omega)$, and therefore there exists only one minimum. ${ }^{1}$ By differentiating Eq. (4) with respect to the real and imaginary part of the control forces $U_{k}(\omega)$ and setting them to zero, it can be shown that the set of the optimum control inputs is the solution of the following linear system of equations

$$
[T(\omega)]\{U(\omega)\}=-F(\omega)\{B(\omega)\},
$$

where vector $\{U(\omega)\}$ contains the $N_{c}$ control inputs, and matrix $[T(\omega)]$ and vector $\{B(\omega)\}$ are defined as 


$$
\begin{aligned}
& {[T(\omega)]} \\
& \quad=\left[\begin{array}{ccc}
\sum_{n=1}^{N} u_{n 1} \xi_{n 1} H_{n}(\omega) & \cdots & \sum_{n=1}^{N} u_{n N_{c}} \xi_{n 1} H_{n}(\omega) \\
\vdots & \ddots & \vdots \\
\sum_{n=1}^{N} u_{n 1} \xi_{n N_{s}} H_{n}(\omega) & \cdots & \sum_{n=1}^{N} u_{n N_{c}} \xi_{n N_{s}} H_{n}(\omega)
\end{array}\right]
\end{aligned}
$$

and

$$
\{B(\omega)\}=\left\{\begin{array}{c}
\sum_{n=1}^{N} f_{n} \xi_{n 1} H_{n}(\omega) \\
\vdots \\
\sum_{n=1}^{N} f_{n} \xi_{n N_{s}} H_{n}(\omega)
\end{array}\right\},
$$

respectively. The elements of matrix $[T(\omega)]$ are the transfer functions between the control inputs and error outputs and has dimension $N_{s} \times N_{c}$, while the elements of vector $\{B(\omega)\}$ are the transfer functions between the disturbance and the error outputs.

Considering Eqs. (4) through (8), the control cost function and the optimum control inputs $U_{k}(\omega)$, are a function of the unit modal control forces $u_{n k}$ and modal error sensor components $\xi_{n s}$. These modal quantities are a measure of the relative controllability and observability of the modes by the actuators and sensors, respectively.

The performance of a feedforward control system will be a function of the type, size, number, and location of the actuators and sensors. This performance can be maximized by optimizing these transducers parameters using formal optimization algorithms. ${ }^{6,7}$ The largest computational effort during the optimization process involves the estimation of the sensitivity of the design variables. In the optimization of feedforward control systems, the design variables are associated to the transducer parameters. Since the control inputs and the error sensors are directly related to the modal control forces and the modal error sensor components, respectively, a sensitivity analysis for these modal parameters is developed here.

\section{SENSITIVITY ANALYSIS}

\section{A. Performance function sensitivity analysis}

As mentioned before, the optimum values for the design variables defining the control system are obtained by minimizing a performance function. This function is in general different from the control cost function defined in Eq. (4). Unlike the control cost function, the performance function will not in general be a quadratic function of the design variables. The average mean-square vibration level over the structure in vibration control and the total structurally radiated power in structural acoustic control are typical examples of the performance function. Thus it is assumed in this work that the performance function can take the form

$$
\Pi\left(u_{n k}, \xi_{n s}\right)=F\left(w(D, \omega), w^{*}(D, \omega)\right),
$$

where $F(\cdot)$ is a linear operator. The performance function $\Pi\left(u_{n k}, \xi_{n s}\right)$ is a function of the control system modal parameters $u_{n k}$ and $\xi_{n s}$. By substituting Eq. (1) into Eq. (9), the performance function can be written as

$$
\Pi\left(u_{n k}, \xi_{n s}\right)=\sum_{m=1}^{N} \sum_{n=1}^{N} q_{m}(\omega) q_{n}^{*}(\omega) F\left(\phi_{m}(D), \phi_{n}(D)\right) .
$$

Now the dependence of the performance function on the design variables $u_{n k}$ and $\xi_{n s}$ lies exclusively on the modal displacements $q_{n}(\omega)$. Equation (10) can be expressed in matrix form as

$$
\Pi\left(u_{n k}, \xi_{n s}\right)=\{q(\omega)\}^{T}[K]\left\{q^{*}(\omega)\right\},
$$

where the modal displacements vector $\{q(\omega)\}$ $=\left\{q_{1}(\omega), \ldots, q_{n}(\omega)\right\}^{T}$ can also be written in matrix form as

$$
\{q(\omega)\}=[H(\omega)]\{\{f\} F(\omega)+[u]\{U(\omega)\}\}
$$

and the $(m, n)$ element of the matrix $[K]$ is

$$
\kappa_{m n}=F\left(\phi_{m}(D), \phi_{n}(D)\right),
$$

which are not a function of the modal parameters $u_{n k}$ and $\xi_{n s}$.

Matrix $[H(\omega)]$ is a diagonal matrix whose elements are the modal frequency response functions defined in Eq. (3), vector $\{f\}=\left\{f_{1}, \ldots, f_{N}\right\}^{T}$ contains the unit modal disturbances, and matrix $[u]$ contains the unit modal control forces where $u_{n k}$ is the $(n, k)$ element of the $N \times N_{c}$ matrix.

As can be seen in Eq. (11) the problem of obtaining the sensitivity of the defined performance function lies in computing the derivatives of the modal displacements with respect to the modal parameters. These parameters arise in Eq. (12) both explicitly and implicitly, through matrix $[u]$ and the control inputs $U_{k}\left(\omega, u_{n k}, \xi_{n s}\right)$, respectively. The implicit dependence of this function with $u_{n k}$ and $\xi_{n s}$ is what increases the complexity of the sensitivity analysis. In this section the sensitivities of the performance function defined in Eq. (11) with respect to these modal parameters are formulated analytically. In order to facilitate the derivation, the analysis is carried out for the modal control forces and the modal error sensor components, separately.

\section{Modal control forces}

The sensitivity of the performance function with respect to the $n$th modal force of the $k$ th control input can be written as

$$
\begin{aligned}
\frac{\partial \Pi\left(u_{n k}, \xi_{n s}\right)}{\partial u_{n k}}= & \{q(\omega)\}^{T}[K]\left\{\frac{\partial q^{*}(\omega)}{\partial u_{n k}}\right\} \\
& +\left\{\frac{\partial q(\omega)}{\partial u_{n k}}\right\}^{T}[K]\left\{q^{*}(\omega)\right\} .
\end{aligned}
$$

The vector involving the partial derivatives of the modal displacements with respect to the unit modal control forces can be expressed as

$$
\left\{\frac{\partial q(\omega)}{\partial u_{n k}}\right\}=\left\{\frac{\partial q(\omega)}{\partial u_{n k}}\right\}_{E}+\left[\frac{\partial q(\omega)}{\partial U(\omega)}\right]\left\{\frac{\partial U(\omega)}{\partial u_{n k}}\right\} .
$$


The first part on the right-hand side of Eq. (15), with the subscript " $E$," contains the explicit differentiation while the second part represents the implicit differentiation. The explicit part is obtained from Eq. (12) as

$$
\left\{\frac{\partial q(\omega)}{\partial u_{n k}}\right\}=U_{k}(\omega) H_{n}(\omega)\left\{e_{n}\right\}
$$

and $\left\{e_{n}\right\}$ is the $n$th standard unit vector where the $n$th element is unity and all the others are zero.

As presented in Eq. (7), vector $\{U(\omega)\}$ is the solution of a linear system of equations. When the number of control inputs equals the number of error sensors, this system can be solved by multiplying on both sides of Eq. (7) by the inverse of matrix $[T(\omega)]$. On the other hand, when there are more error sensors than control inputs the system is solved by the least-squares method. ${ }^{13}$

The least-squares method implies premultiplying Eq. (7) on both sides by the conjugate transpose of matrix $[T(\omega)]$ which yields the Hermitian matrix $[A(\omega)]$ $=[T(\omega)]^{H}[T(\omega)]$, where $H$ implies conjugate transpose. Thus Eq. (7) becomes

$$
[A(\omega)]\{U(\omega)\}=-F(\omega)[T(\omega)]^{H}\{B(\omega)\} .
$$

Having the Hermitian matrix $[A(\omega)]$ instead of matrix $[T(\omega)]$, that is generally non-Hermitian, will generalize the formulation for any control system configuration. For this reason, the premultiplication of Eq. (7) will be adopted regardless if the number of error sensors is equal or greater than the number of control inputs.

The second term in Eq. (15) can now be computed. From Eq. (12) the matrix of the partial derivatives of the modal displacements with respect to the control inputs is

$$
\left[\frac{\partial q(\omega)}{\partial U(\omega)}\right]=[H(\omega)][u] .
$$

In the same way, from Eq. (17), the vector of the partial derivatives of the control inputs with respect to the design variable $u_{n k}$ is expressed as

$$
\begin{aligned}
\left\{\frac{\partial U(\omega)}{\partial u_{n k}}\right\}= & -[A(\omega)]^{-1}\left\{F(\omega)\left[\frac{\partial T(\omega)}{\partial u_{n k}}\right]^{H}\{B(\omega)\}\right. \\
& \left.+\left[\frac{\partial A(\omega)}{\partial u_{n k}}\right]\{U(\omega)\}\right\} .
\end{aligned}
$$

Since the design variable $u_{n k}$ appears in only one column of matrix $[T(\omega)]$, its partial derivatives with respect to $u_{n k}$ yields a matrix full of zeros except for its $k$ th column. Recalling that $[A(\omega)]=[T(\omega)]^{H}[T(\omega)]$, Eq. (19) becomes

$$
\begin{aligned}
\left\{\frac{\partial U(\omega)}{\partial u_{n k}}\right\}= & -[A(\omega)]^{-1}\left(\alpha_{n}(\omega) H_{n}^{*}(\omega)\left\{e_{k}\right\}\right. \\
& \left.+U_{k}(\omega) H_{n}(\omega)[T(\omega)]^{H}\left\{\xi_{n}\right\}\right),
\end{aligned}
$$

where $\left\{\xi_{n}\right\}=\left\{\xi_{n 1}, \xi_{n 2}, \ldots, \xi_{n N s}\right\}^{T}$ is a vector containing the $n$th modal error sensor component for each of the $N_{s}$ error sensors and $\alpha_{n}(\omega)$ is a scalar given as

$$
\alpha_{n}(\omega)=\left\{\xi_{n}\right\}^{T}\{[T(\omega)]\{U(\omega)\}+F(\omega)\{B(\omega)\}\} .
$$

This scalar will vanish for the case when the number of error sensors is equal to the control inputs $\left(N_{c}=N_{s}\right)$. On the other hand, when $N_{s}>N_{c}$, the solution of Eq. (7) is achieved only in a least-square sense, i.e., $[T(\omega)]\{U(\omega)\} \cong-F(\omega)$ $\times\{B(\omega)\}$, and $\alpha_{n}(\omega)$ has a value different from zero.

Finally, Eq. (15) can be written as

$$
\begin{aligned}
\left\{\frac{\partial q(\omega)}{\partial u_{n k}}\right\}= & U_{k}(\omega) H_{n}(\omega)\left\{e_{n}\right\}-[H(\omega)][u] \\
& \times[A(\omega)]^{-1}\left(U_{k}(\omega) H_{n}(\omega)[T(\omega)]^{H}\left\{\xi_{n}\right\}\right. \\
& \left.+H_{n}^{*}(\omega) \alpha_{n}(\omega)\left\{e_{k}\right\}\right) .
\end{aligned}
$$

By substituting this expression into Eq. (14) the sensitivity of the performance function as defined in Eq. (9) with respect to the modal control forces is obtained.

\section{Modal error components}

The sensitivity analysis, with respect to the second set of modal parameters, the modal error sensor components, is implemented in a similar way as for the modal control forces. The partial derivative of the performance function with respect to the $n$th modal component of the $s$ th error sensor can be written as

$$
\begin{aligned}
\frac{\partial \Pi\left(u_{n k}, \xi_{n s}\right)}{\partial \xi_{n s}}= & \{q(\omega)\}^{T}[K]\left\{\frac{\partial q^{*}(\omega)}{\partial \xi_{n s}}\right\} \\
& +\left\{\frac{\partial q(\omega)}{\partial \xi_{n s}}\right\}^{T}[K]\left\{q^{*}(\omega)\right\} .
\end{aligned}
$$

The vector of partial derivatives of the modal displacement with respect to the $n$th modal error sensor of the $s$ th sensor results as

$$
\left\{\frac{\partial q(\omega)}{\partial \xi_{n s}}\right\}=\left[\frac{\partial q(\omega)}{\partial U(\omega)}\right]\left\{\frac{\partial U(\omega)}{\partial \xi_{n s}}\right\} .
$$

Notice that this time there is no explicit part in the differentiation.

Analogous to the modal control forces analysis, the partial derivatives of the control inputs with respect to $\xi_{n s}$ becomes

$$
\begin{aligned}
\left\{\frac{\partial U(\omega)}{\partial \xi_{n s}}\right\}= & -[A(\omega)]^{-1}\left(F(\omega)\left[\frac{\partial T(\omega)}{\partial \xi_{n s}}\right]^{H}\{B(\omega)\}\right. \\
& +F(\omega)[T(\omega)]^{H}\left\{\frac{\partial B(\omega)}{\partial \xi_{n s}}\right\}+\left[\frac{\partial A(\omega)}{\partial \xi_{n s}}\right] \\
& \times\{U(\omega)\}) .
\end{aligned}
$$

Since $\xi_{n s}$ appears in only one component of vector $\{B(\omega)\}$ at a time, its partial derivative with respect to the $n$th modal error component of the $s$ th sensor results in

$$
\left\{\frac{\partial B(\omega)}{\partial \xi_{n s}}\right\}=f_{n} H_{n}\left\{e_{s}\right\}
$$

In the same way, since $\xi_{n s}$ is at one row of matrix $[T(\omega)]$ at a time, the partial derivatives of $[T(\omega)]$ with respect to $\xi_{n s}$ 
becomes a matrix full of zeros except for the $s$ th row. Then Eq. (25) becomes

$$
\begin{aligned}
\left\{\frac{\partial U(\omega)}{\partial \xi_{n s}}\right\}= & -[A(\omega)]^{-1}\left(\beta_{s}(\omega) H_{n}^{*}(\omega)\left\{u_{n}\right\}\right. \\
& \left.+q_{n}(\omega)[T(\omega)]^{H}\left\{e_{s}\right\}\right),
\end{aligned}
$$

where vector $\left\{u_{n}\right\}=\left\{u_{n 1}, u_{n 2}, \ldots, u_{n N c}\right\}^{T}$ contains the $n$th modal control force for each of the $N_{c}$ control inputs and $\beta_{s}(\omega)$ is the $s$ th component of the vector

$$
\{\beta(\omega)\}=\{[T(\omega)]\{U(\omega)\}+F(\omega)\{B(\omega)\}\} .
$$

Then, by substituting Eq. (18) along with Eq. (27) into Eq. (24) it becomes

$$
\begin{aligned}
\left\{\frac{\partial q(\omega)}{\partial \xi_{n s}}\right\}= & -[H(\omega)][u][A(\omega)]^{-1}\left(\beta_{s}(\omega) H_{n}^{*}(\omega)\left\{u_{n}\right\}\right. \\
& \left.+q_{n}(\omega)[T(\omega)]^{H}\left\{e_{s}\right\}\right) .
\end{aligned}
$$

By substituting Eq. (29) into Eq. (23), the sensitivity of the performance function with respect to the modal error components is obtained.

\section{B. Control effort sensitivity analysis}

Another aspect of relevance in the optimum design of feedforward control systems is the control effort, which is a measure of the amount of energy required by the control system. In an optimization scheme, the control effort can be either included in the performance function or as a constraint. The sensitivity of the control effort with respect to the modal parameters can be easily obtained from the results of the previous sections.

In general, the control effort $J_{k}$ for the $k$ th control input $U_{k}(\omega)$ can be defined as

$$
J_{k}=\left|U_{k}(\omega)\right|^{2},
$$

where the brackets imply magnitude. The sensitivity of this function with respect to the modal control forces and the modal error components can be easily obtained by using the formulations already presented in Eqs. (20) and (27), respectively. The sensitivity of the control effort of the $k$ th control input $U_{k}(\omega)$ and $u_{n k}$ can be written as

$$
\frac{\partial J_{k}}{\partial u_{n k}}=U_{k}(\omega) \frac{\partial U_{k}^{*}(\omega)}{\partial u_{n k}}+\frac{\partial U_{k}(\omega)}{\partial u_{n k}} U_{k}^{*}(\omega),
$$

where the partial derivative of the $k$ th control input with respect to $u_{n k}$ is the $k$ th component of the vector defined in Eq. (20). In the same way, the sensitivity of the $k$ th control effort to $\xi_{n s}$ is defined as

$$
\frac{\partial J_{k}}{\partial \xi_{n s}}=U_{k}(\omega) \frac{\partial U_{k}^{*}(\omega)}{\partial \xi_{n s}}+\frac{\partial U_{k}(\omega)}{\partial \xi_{n s}} U_{k}^{*}(\omega)
$$

and the partial derivative of the $k$ th control input with respect to $\xi_{n s}$ is the $k$ th component of the vector defined in Eq. (27).

\section{NUMERICAL EXAMPLE}

The applicability of the formulation presented here is demonstrated for the case of a single input-single output ASAC of a simply supported baffled steel plate harmonically driven by a point disturbance. The plate is assumed to have a Young's modulus $E=2.0 \times 10^{11} \mathrm{~N} / \mathrm{m}^{2}$, density $\rho_{s}=7833$ $\mathrm{Ns}^{2} / \mathrm{m}^{4}$, Poisson's ratio $\nu=0.3$, thickness $h=0.002 \mathrm{~m}$, and dimensions $L_{x}=0.38 \mathrm{~m}$ and $L_{y}=0.30 \mathrm{~m}$. A modal damping ratio of $0.5 \%$ is assumed in all the modes $\left(\beta_{n}=0.005\right)$. Since the analysis is based in the modal representation of the system's response the $n$th natural frequency of the plate is given by

$$
\omega_{n}=\sqrt{\frac{E h^{2}}{12\left(1-\nu^{2}\right) \rho_{s}}}\left[\left(\frac{\pi n_{x}}{L_{x}}\right)^{2}+\left(\frac{\pi n_{y}}{L_{y}}\right)^{2}\right]
$$

and the $n$th mass normalized mode shape has the form

$$
\phi_{n}(x, y)=\sqrt{\frac{4}{L_{x} L_{y} h \rho_{s}}} \sin \left(\frac{\pi n_{x} x}{L_{x}}\right) \sin \left(\frac{\pi n_{y} y}{L_{y}}\right) .
$$

In this example the total acoustic radiated power is selected as the performance function. The acoustic radiated power can be obtained by integrating the acoustic intensity over a half-hemisphere of radius $R$ over the plate. That is

$$
\Pi(\omega)=\int_{0}^{2 \pi} \int_{0}^{\pi / 2} \frac{p(r, \omega) p^{*}(r, \omega)}{\rho_{0} c} R^{2} \sin \theta d \theta d \phi,
$$

where $\rho_{0}$ and $c$ are the density and speed of sound in air, respectively, and $p(r, \omega)$ is the pressure distribution in the far field. The pressure distribution is a linear function of the structural response $w(D, \omega)$. By substituting the response by the modal expansion solution of Eq. (1), the pressure can always be expressed as a summation of the contribution of the pressure produced by each mode as ${ }^{12}$

$$
p(r, \omega)=\sum_{n=1}^{N} q_{n}(\omega) p_{n}(r, \omega) .
$$

For the case of a baffled simply supported plate, the $n$th modal pressure $p_{n}(r, \omega)$ can be obtained by assuming a surface velocity of $j \omega \phi_{n}(D)$ in the Raleigh integral. ${ }^{12}$

By substituting Eq. (36) into Eq. (35) the acoustic radiated power can take the form of Eq. (11), where the matrix $[K]$ contains the acoustic power radiated by the structural modes and its $(m, n)$ component has the form

$\kappa_{m n}(\omega)=\int_{0}^{2 \pi} \int_{0}^{\pi / 2} \frac{p_{m}(r, \omega) p_{n}^{*}(r, \omega)}{\rho_{0} c} R^{2} \sin \theta d \theta d \phi$.

In the example, the sensitivity of the total radiated power with respect to the modal control forces and the modal error sensors is obtained and compared to results computed by the central finite differences technique (CFD) for a range of different perturbations. ${ }^{8}$ Without any loss of generality,

TABLE I. Modal domain data.

\begin{tabular}{cccccc}
\hline $\begin{array}{c}\text { Mode } \\
\text { number } \\
n\end{array}$ & $\begin{array}{c}\text { Modal index } \\
\left(n_{x}, n_{y}\right)\end{array}$ & $\begin{array}{c}\text { Natural } \\
\text { frequency } \\
(\mathrm{Hz})\end{array}$ & $\begin{array}{c}\text { Modal } \\
\text { disturbance } \\
\text { force }\end{array}$ & $\begin{array}{c}\text { Modal } \\
\text { control } \\
\text { force }\end{array}$ & $\begin{array}{c}\text { Modal error } \\
\text { component }\end{array}$ \\
\hline 1 & $(1,1)$ & 86.6 & 1.4837 & 0.7373 & 1.1087 \\
2 & $(2,1)$ & 186.4 & -0.2450 & 0.9983 & 0.1831 \\
3 & $(1,2)$ & 246.8 & -0.3102 & -1.093 & 1.4837 \\
\hline
\end{tabular}




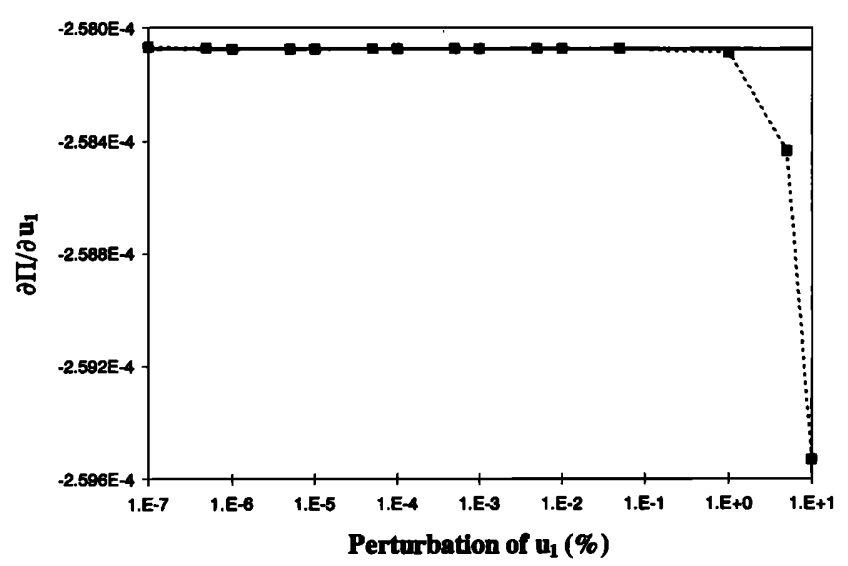

FIG. 2. Sensitivity of radiated power with respect to $u_{1},--$ CFD analytical.

only the first three modes $(N=3)$ are included in the analysis. This small number of modes is enough to compare the proposed analytical formulations to a CFD approximation. A larger number of modes could be easily included without affecting the performance of the analytical formulation. Table I presents the modal indices, natural frequencies, unit modal disturbance forces, unit modal control forces, and the modal error components related to each of the three modes included in the analysis. The disturbance is assumed to have an excitation frequency of $87 \mathrm{~Hz}$ and located at $x_{d}=0.20 \mathrm{~m}$ and $y_{d}=0.16 \mathrm{~m}$. Since there is only one control input and one error sensor, the subscripts $k$ and $s$ are dropped and the modal parameters are expressed as $u_{n}$ and $\xi_{n}$. The modal control forces are obtained assuming the control input to be a point force at $x_{c}=0.10 \mathrm{~m}$ and $y_{c}=0.23 \mathrm{~m}$. The modal error components are obtained assuming the error sensor is a discrete displacement sensor placed at $x_{s}=0.18 \mathrm{~m}$ and $y_{s}=0.08$ $\mathrm{m}$. Therefore, the modal parameters are obtained by computing the value of the mode shapes at the control input and sensor locations.

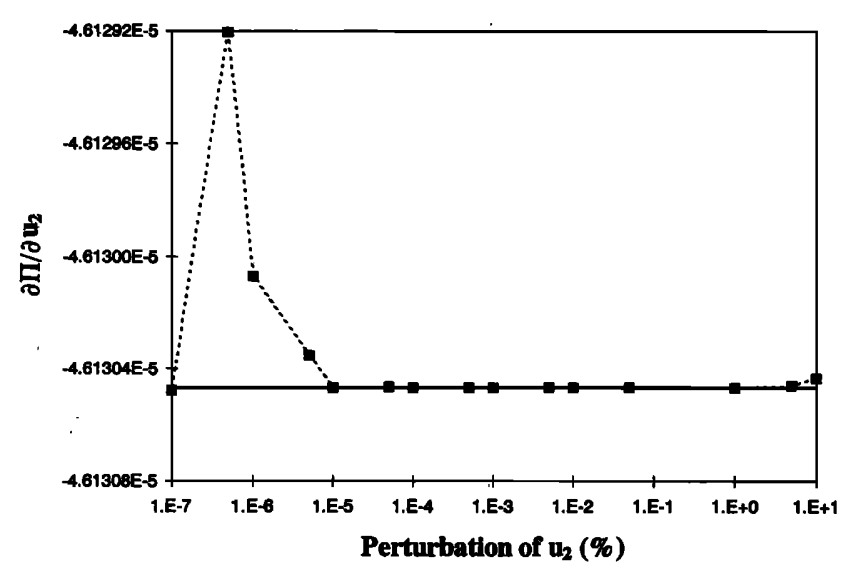

FIG. 3. Sensitivity of radiated power with respect to $u_{2},--$ CFD analytical.

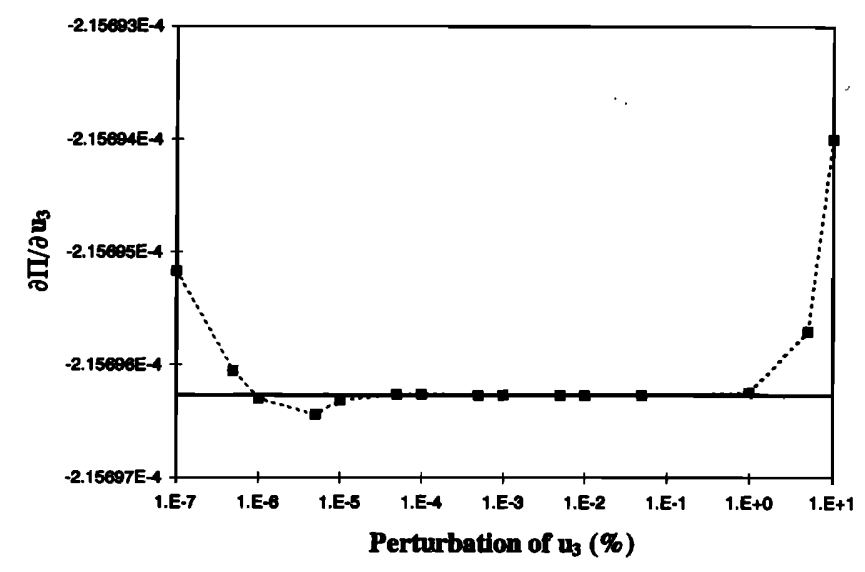

FIG. 4. Sensitivity of radiated power with respect to $u_{3}$, --- CFD analytical.

\section{A. Analytical versus finite differences sensitivities}

Figures 2 through 7 present a comparison in the computation of the sensitivities of the total radiated power $\Pi$ with respect to the modal parameters $u_{n}$ and $\xi_{n}$ by the proposed analytical formulation and the CFD approximations. The CFD approximations are obtained by perturbing the value of each one of the modal parameters by a fraction of its own value. ${ }^{8}$ For example, the approximations in Fig. 2 are obtained using perturbations that range from $\left|u_{1}^{\prime}\right| \times 10^{-9}$ to $\left|u_{1}\right| \times 10^{-1}$.

It is well known that whenever finite differences are used to approximate derivatives, there are two sources of error: truncation and condition errors. ${ }^{9}$ Truncation errors are associated with neglected terms in the Taylor series expansion of the perturbed function and usually occur when the step size in the finite difference computation is too big. This error can be observed in almost all the finite differences results (Figs. 2, 4, and 5) and arise when the perturbations are in the range between $1 \%$ and $10 \%$ of the design variables.

Condition errors are usually related to round-off errors. They arise when the computation involves very small numbers that cannot be correctly represented with the available

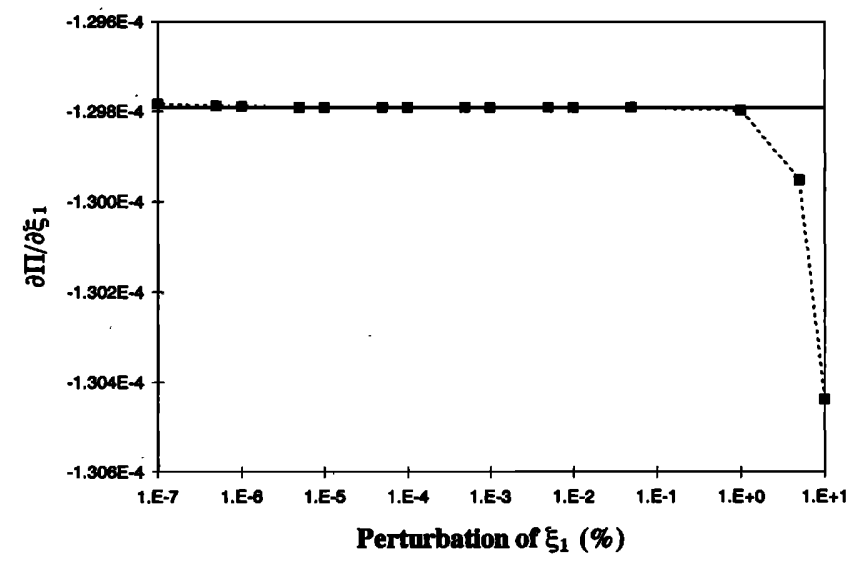

FIG. 5. Sensitivity of radiated power with respect to $\xi_{1},--$ CFD analytical. 


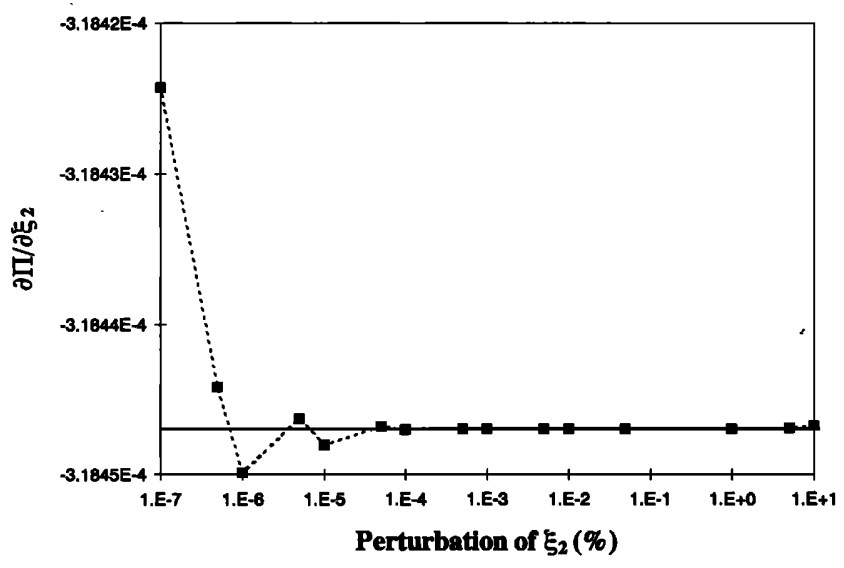

FIG. 6. Sensitivity of radiated power with respect to $\xi_{2},---$ CFD analytical.

significant figures. This type of errors can be observed in the finite differences results for very small perturbations when the sensitivity is related to the second and third mode (Figs. $3,4,6$, and 7). At $87 \mathrm{~Hz}$ the contributions of these modes to the total radiated power, when compared to the first mode, is very small. Therefore finite difference computations related to any of these modes with very small perturbations are sensitive to this type of error. Eventually, if the step size is further reduced, the sensitivity to the modal parameters related to the first mode will be affected as well.

These results show one of the biggest problems of using finite differences, the so called "step size dilemma." If the step size is decreased to reduce the truncation error, it might increase the condition error. To avoid this type of problem is especially important when the sensitivity is going to be implemented into a gradient search algorithm. A minimum error in the sensitivity computation can make the optimization inefficient. This is one of the main advantages of the presented formulation. By having an analytical formulation, the sensitivity will not be affected by these type of errors.

Another significant difference between the two approaches is in terms of the computational time. The average

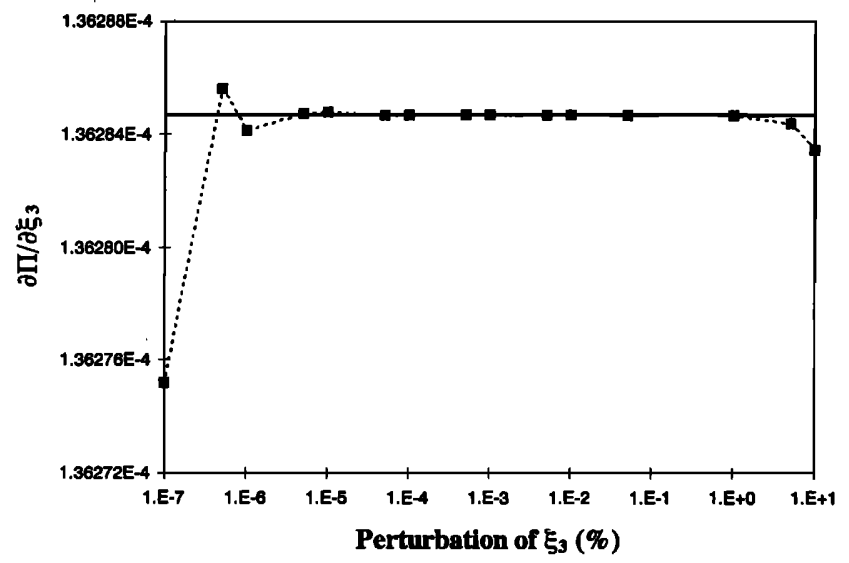

FIG. 7. Sensitivity of radiated power with respect to $\xi_{3},---$ CFD analytical. time for the finite differences computations was $0.25 \mathrm{~s}$, while the average time using the analytical formulation was $0.16 \mathrm{~s}$. That is about $64 \%$ of the time required by the CFD. This aspect becomes critical for the analysis of complex systems with a large number of modes and multiple inputs-multiple outputs control systems.

\section{B. Sensitivity with respect to physical parameters}

As mentioned before, the sensitivity of the performance function $\Pi$ with respect to any of the physical design variables of the control inputs and error sensors can be easily obtained after knowing its partial derivatives with respect to the modal parameters. As an example, the sensitivity of the performance function $\Pi$, with respect of the spatial coordinate $x_{c}$ of the control input $U(\omega)$, can be computed. By using the chain rule of differentiation, this can be expressed as

$$
\frac{\partial \Pi}{\partial x_{c}}=\sum_{n=1}^{N} \frac{\partial \Pi}{\partial u_{n}} \frac{\partial u_{n}}{\partial x_{c}} .
$$

Since the control input is a point force, the modal control parameters $u_{n}$ are defined as the mode shape evaluated at the force location, that is

$$
u_{n}=\phi_{n}\left(x_{c}, y_{c}\right) \text {, }
$$

where $\phi_{n}\left(x_{c}, y_{c}\right)$ is defined in Eq. (34). Then Eq. (38) reduces to

$$
\frac{\partial \Pi}{\partial x_{c}}=\sum_{n=1}^{N} \frac{\partial \Pi}{\partial u_{n}} \frac{\partial \phi_{n}\left(x_{c}, y_{c}\right)}{\partial x_{c}} .
$$

As can be seen from Eq. (38), by using the chain rule of differentiation, the same formulation for the sensitivity of the performance function can be used regardless of the type of transducer. The added computational cost will depend on the expression for the modal parameters for a particular transducer, which is usually a simple function of the physical variables.

\section{CONCLUSIONS}

A formulation for the sensitivity analysis of feedforward finite domain controlled systems with respect to the design parameters of the control inputs and error sensors has been developed. The analysis is performed in the modal domain, which makes the formulation universal for any type of transducer. In this way, the sensitivity with respect to the modal parameters is obtained first, then the sensitivity with respect to any physical design variable of a particular transducer can be found using the chain rule of differentiation. By using an analytical approach, the computation of the sensitivities, with respect to the modal parameters, is reduced into a set of simple calculations that can be easily implemented into a formal optimization procedure. Most parts of the formulation consist in matrix multiplications. The major computational effort lies in solving the inversion of a Hermitian matrix, which has to be done only once. This matrix is of the size of the number of control inputs $\left(N_{c} \times N_{c}\right)$, which is usually a small number. Results from this approach were compared in an example to the ones obtained by using the finite difference 
technique. In the example it was demonstrated that the proposed formulation provides a better solution in terms of accuracy and computing time. Both of these aspects are of major concern when the sensitivities are intended to be used along with formal optimization algorithms.

\section{ACKNOWLEDGMENT}

The authors gratefully acknowledge the support of this work by the Office of Naval Research Grant No. ONRN00014-92-J-1170.

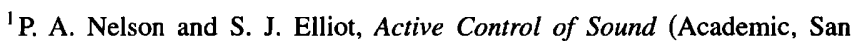
Diego, CA, 1993).

${ }^{2}$ C. R. Fuller, "Active Control of Sound Transmission/Radiation from Elastic Plates by Vibrating Inputs-I Analysis," J. Sound Vib. 136(1), 1-15 (1990).

${ }^{3}$ V. L. Metcalf, C. R. Fuller, R. J. Silcox, and D. E. Brown, “Active Control of Sound Transmission/Radiation from Elastic Plates by Vibrating Inputs-II Experiments," J. Sound Vib. 153(3), 387-401 (1992).

${ }^{4}$ R. L. Clark and C. R. Fuller, "Experiments on Active Control of Structurally Radiated Sound Using Multiple Piezoceramic Actuators," J. Acoust. Soc. Am. 91, 3313-3320 (1992).
${ }^{5}$ C. R. Fuller, G. P. Gibbs, and R. J. Silcox, "Simultaneous active control of flexural and extensional power flow in beams," J. Intelligent Mater. Syst. Structures 1(2), 235-247 (1990).

${ }^{6}$ B. T. Wang, R. A. Burdisso, and C. R. Fuller, "Optimal Placement of Piezoelectric Actuators for Active Control of Sound Radiation from Elastic Plates," in Proceedings of Noise-Conference 91 (Noise Control Foundation, New York, 1991), pp. 267-275.

${ }^{7}$ R. L. Clark and C. R. Fuller, "Optimal Placement of Piezoelectric Actuators and Polyvinylidene Fluoride (PVDF) Error Sensors in Active Structural Acoustic Control Approaches," J. Acoust. Soc. Am. 92, 1521-1533 (1992).

${ }^{8}$ J. S. Arora, Introduction to Optimum Design (McGraw-Hill, New York, 1989).

${ }^{9}$ R. A. Haftka and Z. Gurdal, Elements of Structural Optimization (Kluwer Academic, Norwell, MA, 1992).

${ }^{10}$ E. K. Dimitriadis, C. R. Fuller, and C. R. Rogers, "Piezoelectric Actuators for Distributed Vibration Excitation of Thin Plates," J. Vib. Acoust. 113, 100-107 (1991).

${ }^{11}$ R. A. Burdisso and C. R. Fuller, "Theory of Feedforward Controlled System Eigenproperties," J. Sound Vib. 153(3), 437-452 (1992).

${ }^{12}$ R. A. Burdisso and C. R. Fuller, "Dynamic Behavior of StructuralAcoustic Systems in Feedforward Control of Sound Radiation," J. Acoust. Soc. Am. 92(1), 277-286 (1992).

${ }^{13}$ S. C. Chapra and R. P. Canale, Numerical Methods for Engineers (McGraw-Hill, New York, 1988). 